Pure and Applied Mathematics Quarterly

Volume 4, Number 4

(Special Issue: In honor of

Jean-Pierre Serre, Part 1 of 2)

$1215-1232,2008$

\title{
Geometrically Connected Components of Lubin-Tate Deformation Spaces with Level Structures
}

\section{Matthias Strauch}

Dedicated to Jean-Pierre Serre on the occasion of his $80^{\text {th }}$ birthday

\begin{abstract}
We determine the geometrically connected components of the generic fibre of the deformation space $\mathcal{M}_{m}$ which parameterizes deformations of a one-dimensional formal o-module equipped with Drinfeld level$m$-structures. It is shown that the geometrically connected components are defined over a Lubin-Tate extension of the base field, and the action of the covering group $G L_{n}\left(\mathfrak{o} / \varpi^{m}\right)$ ) on the components is given by the determinant. This furnishes a description of the action of this group on the étale cohomology of the spaces $\mathcal{M}_{m}^{\text {rig }}$ in degree zero.
\end{abstract}

\section{Contents}

1. Introduction 1215

2. Preliminaries 1218

3. The Galois action on the determinant of the Tate module 1221

4. Geometrically connected components and the group action on $\pi_{0} \quad 1224$

$\begin{array}{ll}\text { References } & 1231\end{array}$

\section{INTRODUCTION}

Let $F$ be a local non-Archimedean field, and denote by $\mathfrak{o}$ its ring of integers. Let $\mathbb{X}$ be a one-dimensional formal $\mathfrak{o}$-module of $F$-height $n$ over the algebraic closure

Received August 11, 2006. 
$\mathbb{F}$ of the residue field of $\mathfrak{o}$. Generalizing work of Lubin and Tate, cf. [LT], V. G. Drinfeld showed in $[\mathrm{D}]$ that the functor of deformations of $\mathbb{X}$ is representable by an affine formal scheme $\mathcal{M}_{0}=\operatorname{Spf}(R)$, where $R \simeq \hat{\mathfrak{o}}^{n r}\left[\left[u_{1}, \ldots, u_{n-1}\right]\right]$. Here, $\hat{\mathfrak{o}}^{n r}$ is the completion of the maximal unramified extension of $\mathfrak{o}$. Moreover, Drinfeld introduced the notion of a level- $m$-structure, and proved that the functor of deformations of $\mathbb{X}$ which are equipped with a level- $m$-structure is representable by a formal scheme $\mathcal{M}_{m}=\operatorname{Spf}\left(R_{m}\right)$, where $R_{m}$ is a regular local ring which is a finite flat $R$-module, and étale over $R$ after inverting a uniformizer $\varpi$ of $\mathfrak{o}$. In this paper we are interested in the geometrically connected components of the rigid-analytic space

$$
M_{m}=\mathcal{M}_{m}^{r i g}
$$

associated to $\mathcal{M}_{m}$, cf. [dJ2], sec. 7. The étale cohomology groups of the spaces $M_{m}$ have been investigated in the last two decades by various authors because of their significance for the local Langlands correspondence. Let us cite only [Ca], $[\mathrm{Bo}]$, and $[\mathrm{HT}]$. According to conjectures by Carayol and Drinfeld, the inductive limit

$$
H^{n-1}=\lim _{\vec{m}} H^{n-1}\left(M_{m} \times_{\hat{F}^{n r}} \mathbb{C}_{\varpi}, \overline{\mathbb{Q}_{l}}\right)
$$

realizes simultaneously the Jacquet-Langlands and the Langlands correspondence (cf. [Ca] for a more precise statement). Here $\hat{F}^{n r}$ is the field of fractions of $\hat{\mathfrak{o}}^{n r}$, and $\mathbb{C}_{\varpi}$ is the completion of an algebraic closure of $\hat{F}^{n r}$.

Whereas the spaces $M_{m}$ are defined purely locally, the analysis of the inductive limit above is carried out in [Bo] and [HT] by embedding the local situation into a global one. This is done because it is very hard to understand the action of the inertia group on $H^{n-1}$ (however, there are results for $m=1$ or $n=2$, cf. [Y] resp. [W]). By studying the geometry of the spaces $M_{m}$ purely locally, it is possible to understand the action of the pro-covering group $G L_{n}(\mathfrak{o})$ and the action of Aut $(\mathbb{X})$, thereby proving the assertion concerning the Jacquet-Langlands correspondence (cf. [St], where the results are unconditionally proved for the Euler-Poincaré characteristic of the cohomology). Of course, one would like to understand explicitly the cohomology in all degrees, and the most basic task is hence to determine the representation on $H^{0}\left(M_{m} \times \hat{F}^{n r} \mathbb{C}_{\varpi}, \overline{\mathbb{Q}_{l}}\right)$. This means to identify the group action on the set of geometrically connected components of $M_{m}$. 
We are going to explain how we do this. For $m \geq 0$ put $K_{m}=\operatorname{Frac}\left(R_{m}\right)$, $K=K_{0}$, and fix a separable closure $K^{s}$ of $K$ containing all $K_{m}$. Denote by $G_{K}=\operatorname{Gal}\left(K^{s} / K\right)$ the absolute Galois group of $K$. Let $X^{\text {univ }}$ be the universal deformation of $\mathbb{X}$ over $R$, and let

$$
T_{\varpi m}=X^{u n i v}\left[\varpi^{m}\right]\left(K^{s}\right)
$$

be the group of $K^{s}$-valued points of the $\varpi^{m}$-torsion subgroup of $X^{\text {univ }} . T_{\varpi m}$ is free of rank $n$ over $\mathfrak{o} /\left(\varpi^{m}\right)$. Then we show, exactly as Raynaud in $[\mathrm{R}]$, that the induced action of $G_{K}$ on $\Lambda^{n}\left(T_{\varpi^{m}}\right)$ factorizes through the canonical isomorphism

$$
\operatorname{Gal}\left(\hat{F}_{m}^{n r} / \hat{F}^{n r}\right) \longrightarrow\left(\mathfrak{o} /\left(\varpi^{m}\right)\right)^{\times}
$$

where $\hat{F}_{m}^{n r} \subset K^{s}$ is obtained from $\hat{F}^{n r}$ by adjoining all $\varpi^{m}$-torsion points of a fixed formal o-module $L T$ of height one over $\hat{\mathfrak{o}}^{n r}$. As $\operatorname{Gal}\left(K^{s} / K_{m}\right)$ acts trivially on $T_{\varpi m}$, and hence on $\Lambda^{n}\left(T_{\varpi^{m}}\right)$, this implies that $\hat{F}_{m}^{n r}$ is contained in $K_{m}$. Then one shows that $R_{m} / \varpi_{m}$ is reduced, where $\varpi_{m}$ is a uniformizer of $\hat{F}_{m}^{n r}$, and using a result of de Jong, cf. [dJ2], 7.3.5, one obtains that $M_{m}$ is geometrically connected over $\hat{F}_{m}^{n r}$.

When thinking about this problem of geometrically connected components we were inspired by de Jong's paper [dJ1]. De Jong also uses the crucial fact that the action on the determinant of the Tate module is given by the cyclotomic character (he considers only the case $F=\mathbb{Q}_{p}$ ), but his further reasoning is different from ours as he is interested in a description of the étale fundamental group of the corresponding period space, cf. [dJ1], Prop. 7.4.

We conclude with a remark on an earlier approach to the problem of geometrically connected components of these spaces. Let $\hat{\mathfrak{o}}_{m}^{n r}$ be the ring of integers of the Lubin-Tate extension $\hat{F}_{m}^{n r}$. From the inclusion $\hat{\mathfrak{o}}_{m}^{n r} \subset R_{m}$ we get a morphism of formal schemes

$$
\mathcal{M}_{m} \longrightarrow \mathcal{M}_{m}^{(1)}:=\operatorname{Spf}\left(\mathfrak{o}_{\hat{F}_{m}^{n r}}\right)
$$

$\mathcal{M}_{m}^{(1)}$ can be intrinsically defined as the deformation space with level- $m$-structures of the reduction $L T_{\mathbb{F}}$ of $L T$. Our original aim was to define a priori a functorial map like this, and then to deduce that $M_{m}$ is geometrically connected over $\left(\mathcal{M}_{m}^{(1)}\right)^{r i g}=\operatorname{Sp}\left(\hat{F}_{m}^{n r}\right)$. A functorial map as above can be thought of as associating to a deformation of $\mathbb{X}$ which is equipped with a level- $m$-structure: 


$$
\left(X,\left(\varpi^{-m} \mathfrak{o} / \mathfrak{o}\right)^{n} \stackrel{\phi}{\longrightarrow} X\left[\varpi^{m}\right]\right)
$$

its determinant:

$$
\left(\Lambda^{n}(X), \Lambda^{n}\left(\varpi^{-m} \mathfrak{o} / \mathfrak{o}\right)^{n} \stackrel{\Lambda^{n} \phi}{\longrightarrow} \Lambda^{n}(X)\left[\varpi^{m}\right]\right)
$$

In an unpublished manuscript J. Lubin treats the problem of defining the determinant of a one-dimensional formal module together with a determinant map, cf. [L], and we, in our earlier approach, worked along the same lines. First one has to define $\Lambda^{n}(X)$, what one may do using, for example, Zink's theory of displays (resp., in the equal characteristic case, the 'module des coordonnées', cf. [Bo]). Then, and more difficult it seems, one has to compare the Tate modules. Using Falting's results in [F], cf. p. 278, one may possibly do this. The approach of this paper, however, seems to be much more elementary.

Acknowledgements. I would like to thank Professor J. Lubin for sending me his unpublished manuscript on determinants of formal groups. Moreover, I thank L. Fargues for very helpful discussions on the subject of this paper.

Notation. In this paper, $F$ will be a non-Archimedean local field, with ring of integers $\mathfrak{o}$, and $\varpi$ will be a uniformizer of $F$. The number of elements of the residue field will be denoted by $q$, and the residue field itself by $\mathbb{F}_{q}$. We denote by $\mathbb{F}$ an algebraic closure of $\mathbb{F}_{q} . \hat{F}^{n r}$ is the completion of the maximal unramified extension of $F$, and $\hat{\mathfrak{o}}^{n r}$ its ring of integers. $\mathbb{C}_{\varpi}$ denotes a the completion of an algebraic closure of $\hat{F}^{n r}$. If $A$ is a local ring we denote by $\mathfrak{m}_{A}$ its maximal ideal. The residue field of a point $x$ on a scheme will be denoted by $\kappa(x)$.

\section{Preliminaries}

In this section we recall without proof some facts about the formal deformation schemes $\mathcal{M}_{m}$, cf. [D], [St].

2.1. Let $\mathbb{X}$ be a one-dimensional formal group over $\mathbb{F}$ that is equipped with an action of $\mathfrak{o}$, i.e. we assume given a homomorphism $\mathfrak{o} \rightarrow \operatorname{End}_{\mathbb{F}}(\mathbb{X})$ such that the action of $\mathfrak{o}$ on the tangent space is given by the reduction map $\mathfrak{o} \rightarrow \mathbb{F}_{q} \subset \mathbb{F}$. Such an object is called a formal $\mathfrak{o}$-module over $\mathbb{F}$. Moreover, we assume that $\mathbb{X}$ is of $F$-height $n$, which means that the kernel of multiplication by $\varpi$ is a finite group 
scheme of rank $q^{n}$ over $\mathbb{F}$.

It is known that for each $n \in \mathbb{Z}_{>0}$ there exists a formal $\mathfrak{o}$-module of $F$-height $n$ over $\mathbb{F}$, and that it is unique up to isomorphism [D], Prop. 1.6, 1.7.

Let $\mathcal{C}$ be the category of complete local noetherian $\hat{\mathfrak{o}}^{n r}$-algebras with residue field $\mathbb{F}$. A deformation of $\mathbb{X}$ over an object $A$ of $\mathcal{C}$ is a pair $(X, \iota)$, consisting of a formal o-module $X$ over $A$ which is equipped with an isomorphism $\iota: \mathbb{X} \rightarrow X_{\mathbb{F}}$ of formal o-modules over $\mathbb{F}$, where $X_{\mathbb{F}}$ denotes the reduction of $X$ modulo the maximal ideal $\mathfrak{m}_{A}$ of $A$. Sometimes we will omit $\iota$ from the notation.

Following Drinfeld $[\mathrm{D}]$, sec. 4B, we define a structure of level $m$ or level-mstructure on a deformation $X$ over $A \in \mathcal{C}(m \geq 0)$ as an $\mathfrak{o}$-module homomorphism

$$
\phi:\left(\varpi^{-m} \mathfrak{o} / \mathfrak{o}\right)^{n} \longrightarrow \mathfrak{m}_{A},
$$

such that, after having fixed a coordinate $T$ on the formal group $X$, the power series $[\varpi]_{X}(T) \in A[[T]]$, which describes the multiplication by $\varpi$ on $X$, is divisible by

$$
\prod_{a \in\left(\varpi^{-1} \mathfrak{o} / \mathfrak{o}\right)^{n}}(T-\phi(a))
$$

Here, $\mathfrak{m}_{A}$ is given the structure of an $\mathfrak{o}$-module via $X$.

Define the following set-valued functor $\mathcal{M}_{m}$ on the category $\mathcal{C}$. For an object $A$ of $\mathcal{C}$ put

$\mathcal{M}_{m}(A)=\{(X, \iota, \phi) \mid(X, \iota)$ is a def. over $A, \phi$ is a level-m-structure on $X\} / \simeq$,

where $(X, \iota, \phi) \simeq\left(X^{\prime}, \iota^{\prime}, \phi^{\prime}\right)$ if and only if there is an isomorphism $(X, \iota) \rightarrow$ $\left(X^{\prime}, \iota^{\prime}\right)$ of formal o-modules over $A$, which is compatible with the level structures. For $0 \leq m^{\prime} \leq m$ one gets by restricting a level- $m$-structure to

$$
\left(\varpi^{-m^{\prime}} \mathfrak{o} / \mathfrak{o}\right)^{n} \subset\left(\varpi^{-m} \mathfrak{o} / \mathfrak{o}\right)^{n}
$$

a level- $m^{\prime}$-structure and hence a natural transformation 


$$
\mathcal{M}_{m} \longrightarrow \mathcal{M}_{m^{\prime}}
$$

Put $\mathfrak{o}_{B}=\operatorname{End}_{\mathfrak{o}}(\mathbb{X}) . \mathfrak{o}_{B}$ is the ring of integers in a central division algebra over $F$ with Hasse invariant $\frac{1}{n}$ ([D], Prop. 1.7). There is a natural action of $G L_{n}\left(\mathfrak{o} / \varpi^{m}\right) \times \mathfrak{o}_{B}^{\times}$from the right on the functor $\mathcal{M}_{m}$ given by

$$
[X, \iota, \phi] \cdot(g, b)=[X, \iota \circ b, \phi \circ g]
$$

where $(g, b) \in G L_{n}\left(\mathfrak{o} / \varpi^{m}\right) \times \mathfrak{o}_{B}^{\times}$and $[X, \iota, \phi]$ denotes the equivalence class of $(X, \iota, \phi)$.

Theorem 2.2. (i) The functor $\mathcal{M}_{m}$ is representable by a regular local ring $R_{m}$ of dimension $n$. Hence there is a universal formal $\mathfrak{o}$-module $X^{\text {univ }}$ over $R:=R_{0}$ which defines on the maximal ideal $\mathfrak{m}_{R_{m}}$ of $R_{m}$ the structure of an $\mathfrak{o}$-module. There is a universal level-m-structure

$$
\phi_{m}^{\text {univ }}:\left(\varpi^{-m} \mathfrak{o} / \mathfrak{o}\right)^{n} \longrightarrow \mathfrak{m}_{R_{m}}
$$

such that, if $a_{1}, \ldots, a_{n}$ is a basis of $\left(\varpi^{-m} \mathfrak{o} / \mathfrak{o}\right)^{n}$ over $\mathfrak{o} /\left(\varpi^{m}\right)$, then

$$
\phi_{m}^{\text {univ }}\left(a_{1}\right), \ldots, \phi_{m}^{u n i v}\left(a_{n}\right)
$$

is a regular system of parameters for $R_{m}$.

(ii) The ring homomorphism $R_{m} \rightarrow R$ which corresponds to the natural transformation $\mathcal{M}_{m} \rightarrow \mathcal{M}_{0}$ makes $R_{m}$ a finite and flat $R$-algebra. Moreover, $R_{m}\left[\frac{1}{\varpi}\right]$ is étale and galois over $R\left[\frac{1}{\varpi}\right]$ with Galois group isomorphic to $G L_{n}\left(\mathfrak{o} / \varpi^{m}\right)$.

(iii) $R$ is (non-canonically) isomorphic to $\hat{\mathfrak{o}}^{n r}\left[\left[u_{1}, \ldots, u_{n-1}\right]\right]$.

Proof. (i) This result is [D], Prop. 4.3.

(ii) That $R_{m}$ is finite and flat over $R$ is again [D], Prop. 4.3. For the second statement we refer to [St], Thm. 2.1.2.

(iii) This is $[\mathrm{D}]$, Prop. 4.2 .

Remark. The fact that $\hat{\mathfrak{o}}^{n r}\left[\left[u_{1}, \ldots, u_{n-1}\right]\right]$ represents $\mathcal{M}_{0}$ is due to Lubin and Tate (for $F=\mathbb{Q}_{p}$ ), cf. [LT]. For this reason $\mathcal{M}_{0}$, the deformation space without 
level structures, is sometines called the Lubin-Tate moduli space, cf. [HG], [Ch].

By the preceding theorem, $R_{m}$ is a domain, and we put $K_{m}=\operatorname{Frac}\left(R_{m}\right)$. This is a Galois extension of $K:=K_{0}$ with Galois group canonically isomorphic to $G L_{n}\left(\mathfrak{o} / \varpi^{m}\right)$. The maps $\mathcal{M}_{m} \rightarrow \mathcal{M}_{m^{\prime}}$ induce injections $K_{m^{\prime}} \hookrightarrow K_{m}$. Put

$$
K_{\infty}=\cup_{m \geq 0} K_{m}
$$

and fix a separable closure $K^{s}$ of $K$ containing $K_{\infty}$.

2.3. We conclude this section by recalling that one may choose the parameters $\varpi=u_{0}, u_{1}, \ldots, u_{n-1}$ of $R$ and the coordinate on $X^{\text {univ }}$ such that multiplication by $\varpi$ on $X^{\text {univ }}$ is given by a power series $[\varpi]_{X^{\text {univ }}}(T) \in R[[T]]$ with the property that

$$
[\varpi]_{X^{u n i v}}(T) \equiv u_{i} T^{q^{i}} \bmod \left(u_{0}, \ldots, u_{i-1}\right), \operatorname{deg}\left(q^{i}+1\right)
$$

cf. [HG], Prop. 5.7. In particular, if $x \in \operatorname{Spec}(R)$ is a point where $\varpi$ vanishes but $u_{1}$ is invertible in the residue field $\kappa(x)$ of $x$, the multiplication of $\varpi$ on the formal group $X^{\text {univ }} \hat{\otimes} \kappa(x)$ has as kernel a group scheme of order $q$. Therefore the connected component of the associated $\varpi$-divisible group $\left(X^{\text {univ }}\left[\varpi^{\infty}\right]\right) \otimes \kappa(x)$ over $\kappa(x)$ is a formal $\mathfrak{o}$-module of height one.

\section{The Galois action on the Determinant of the Tate module}

3.1. Denote by $T$ the Tate module of the $\varpi$-divisible group $X^{\text {univ }}\left[\varpi^{\infty}\right] \otimes K$ :

$$
T=\lim _{\overleftarrow{m}} X^{u n i v}\left[\varpi^{m}\right]\left(K^{s}\right)
$$

The universal Drinfeld level-structures furnish an isomorphism of $\mathfrak{o}$-modules

$$
\mathfrak{o}^{n} \longrightarrow T
$$

so that $\Lambda_{\mathfrak{o}}^{n}(T)$ is free of rank one over $\mathfrak{o}$. 
3.2. We recall some facts from Lubin-Tate theory. Fix a formal o-module $L T$ of height one over $\hat{\mathfrak{o}}^{n r}$. As the universal deformation ring of height one formal $\mathfrak{o}$ modules is just $\hat{\mathfrak{o}}^{n r}$, all such formal $\mathfrak{o}$-modules are isomorphic. Denote by $\hat{F}^{n r, s} \subset$ $K^{s}$ the algebraic closure of $\hat{F}^{n r}$ in $K^{s}$. It is a separable closure of $\hat{F}^{n r}$. Let $\mathfrak{m}$ be the maximal ideal of the ring of integers in the completion of $\hat{F}^{n r, s} \cdot \mathfrak{m}$ is equipped via $L T$ with an o-module structure, and the torsion points of $L T$ in $\mathfrak{m}$ are known to lie in $\hat{F}^{n r, s}$. Let $\hat{F}_{m}^{n r} \subset \hat{F}^{n r, s}$ be the subfield generated over $\hat{F}^{n r}$ by the $\varpi^{m}$-torsion points of $L T$ in $\mathfrak{m}$. As all $\mathfrak{o}$-modules of height one over $\hat{\mathfrak{o}}^{n r}$ are isomorphic over $\hat{\mathfrak{o}}^{n r}$, this field is independent of the choice of $L T$. There is a canonical isomorphism

$$
\chi_{m}: \operatorname{Gal}\left(\hat{F}_{m}^{n r} / \hat{F}^{n r}\right) \longrightarrow\left(\mathfrak{o} /\left(\varpi^{m}\right)\right)^{\times},
$$

such that for any $\varpi^{m}$-torsion point $\alpha$ of $L T$ and $\sigma \in \operatorname{Gal}\left(\hat{F}_{m}^{n r} / \hat{F}^{n r}\right)$ one has

$$
\sigma(\alpha)=\left[\chi_{m}(\sigma)\right]_{L T}(\alpha)
$$

The field $\hat{F}_{\infty}^{n r}=\bigcup_{m} \hat{F}_{m}^{n r}$ is the maximal abelian extension of $\hat{F}^{n r}$. The characters $\chi_{m}$ induce an isomorphism

$$
\chi: \operatorname{Gal}\left(\hat{F}_{\infty}^{n r} / \hat{F}^{n r}\right) \longrightarrow \mathfrak{o}^{\times},
$$

Finally, let $\tilde{\chi}: G_{K} \rightarrow \mathfrak{o}^{\times}$be the composition of $G_{K} \rightarrow \operatorname{Gal}\left(\hat{F}_{\infty}^{n r} / \hat{F}^{n r}\right)$ with $\chi$.

The following theorem is essentially Raynaud's theorem on the action of the Galois group on the determinant of the Tate module of a $p$-divisible group, cf. $[\mathrm{R}]$, Thm. 4.2.1. We prove it here again for the sake of completeness and because we need it in the more general context of formal $\mathfrak{o}$-modules.

Theorem 3.3. The natural action of $G_{K}=\operatorname{Gal}\left(K^{s} / K\right)$ on $\Lambda_{\mathfrak{o}}^{n}(T)$ is given by the character $\tilde{\chi}$, i.e. for all $\sigma \in G_{K}$ and $\lambda \in \Lambda_{\mathfrak{o}}^{n}(T)$ one has:

$$
\sigma(\lambda)=\tilde{\chi}(\sigma) \lambda
$$

Proof. We assume $n \geq 2$, because for $n=1$ everything is trivial. We follow the reasoning of the proof of $[\mathrm{R}]$, Th. 4.2.1. Let $x \in \operatorname{Spec}(R)$ be a prime ideal containing $\varpi$ but not containing $u_{1}$, and denote by $\kappa(x)$ the residue field at $x$. We already noticed in 2.3 that the connected component of the $\varpi$-divisible group 
$X^{\text {univ }}\left[\varpi^{\infty}\right] \otimes \kappa(x)$ is a formal $\mathfrak{o}$-module of height one, and the étale part is hence of height $n-1$. Let $R_{x}^{s h}$ be the strict henselization of $R$ at $x$. Its residue field $\kappa(x)^{s}$ is a separable closure of $\kappa(x)$. By [D], Prop. 1.7, all formal o-modules of finite height over a separably closed field are isomorphic, so that the formal module associated to the connected component

$$
\left(X^{u n i v}\left[\varpi^{\infty}\right] \otimes \kappa(x)^{s}\right)^{\circ}
$$

is isomorphic to $L T \hat{\otimes}_{\hat{\mathfrak{o}} n r} \kappa(x)^{s}$. Therefore, the formal module associated to

$$
\left(X^{u n i v}\left[\varpi^{\infty}\right] \otimes R_{x}^{s h}\right)^{\circ}
$$

is isomorphic to a deformation of $L T \hat{\otimes}_{\hat{\mathfrak{o}}^{n r}} \kappa(x)^{s}$, hence it is isomorphic to

$$
L T \hat{\otimes}_{\hat{\mathfrak{o}}^{n r}} R_{x}^{s h}
$$

because there is up to isomorphism only one deformation, cf. [Ha], Thm. 22.4.16. Let $R_{x}^{h} \subset R_{x}^{s h}$ be the henselization of $R$ at $x$, put $K_{x}^{h}=\operatorname{Frac}\left(R_{x}^{h}\right)$, and fix a separable closure $K_{x}^{h, s}$ of $K_{x}^{h}$ together with an embedding of $K^{s}$ into this separable closure. We get an embedding of Galois groups $\operatorname{Gal}\left(K_{x}^{h, s} / K_{x}^{h}\right) \hookrightarrow G_{K}$, and it follows from what we have said above that the restriction of the $G_{K}$-action on

$$
\Pi:=\Lambda_{\mathfrak{o}}^{n}(T) \otimes \tilde{\chi}^{-1}
$$

to $\operatorname{Gal}\left(K_{x}^{h, s} / K_{x}^{h}\right)$ factors through the absolute Galois group of $\kappa(x)$. That means, the representation of $G_{K}$ on $\Pi$ is unramified at $x$. As the $\varpi$-divisible group $X^{\text {univ }}\left[\varpi^{\infty}\right]$ is étale at every point of $\operatorname{Spec}(R)$ where $\varpi$ is invertible, $\Pi$ is unramified at all points of $U=\operatorname{Spec}(R)-V\left(\left(\varpi, u_{1}\right)\right)$, whose complement is of codimension 2. Hence the representation of $G_{K}$ on $\Pi$ extends to the étale fundamental group $\pi_{1}\left(U, \operatorname{Spec}\left(K^{s}\right)\right)$ of $U$. But by the Zariski-Nagata purity theorem, cf. [SGA2], exp. X, Thm. 3.4, [SGA1], exp. X, Cor. 3.3, $\pi_{1}\left(U, \operatorname{Spec}\left(K^{s}\right)\right)=\pi_{1}\left(\operatorname{Spec}(R), \operatorname{Spec}\left(K^{s}\right)\right)$, and the latter group is trivial, as $R$ is strictly henselian. This means that $G_{K}$ acts trivially on $\Pi$ and therefore by $\tilde{\chi}$ on $\Lambda_{\mathfrak{o}}^{n}(T)$.

Corollary 3.4. (i) The action of $G_{K}$ on $\Lambda_{\mathfrak{o}}^{n}\left(X^{\text {univ }}\left[\varpi^{m}\right]\left(K^{s}\right)\right)$ is given by the character $\tilde{\chi}_{m}$, which is the composition of $\tilde{\chi}$ with the canonical map $\mathfrak{o}^{\times} \rightarrow$ $\left(\mathfrak{o} / \varpi^{m}\right)^{\times}$. 
(ii) The ring $R_{m}=\mathcal{O}\left(\mathcal{M}_{m}\right)$ contains the ring of integers $\hat{\mathfrak{o}}_{m}^{n r}$ of the Lubin-Tate extension $\hat{F}_{m}^{n r}$.

Proof. (i) This assertion follows immediately from the $G_{K}$-equivariant isomorphism

$$
\Lambda_{\mathfrak{o}}^{n}\left(X^{u n i v}\left[\varpi^{m}\right]\left(K^{s}\right)\right) \simeq \Lambda_{\mathfrak{o}}^{n}(T) / \varpi^{m} \Lambda_{\mathfrak{o}}^{n}(T)
$$

(ii) The subgroup $\operatorname{Gal}\left(K^{s} / K_{m}\right) \subset G_{K}$ acts trivially on $X^{\text {univ }}\left[\varpi^{m}\right]\left(K^{s}\right)$, and hence trivially on $\Lambda^{n}\left(X^{\text {univ }}\left[\varpi^{m}\right]\left(K^{s}\right)\right)$. By (i) the action on the latter module is given by $\tilde{\chi}_{m}$. Therefore, $\operatorname{Gal}\left(K^{s} / K_{m}\right)$ acts trivially on $\hat{F}_{m}^{n r}$, and hence $\hat{F}_{m}^{n r}$ is contained in $K_{m}$. Because $R_{m}$ is integrally closed, it contains $\hat{\mathfrak{o}}_{m}^{n r}$.

By the second assertion, we will view from now on $R_{m}$ as an $\hat{\mathfrak{o}}_{m}^{n r}$-algebra, and $R_{m}\left[\frac{1}{\varpi}\right]$ as an $\hat{F}_{m}^{n r}$-algebra.

\section{Geometrically connected components and the group action on}

$\pi_{0}$

4.1. Fix an integer $m \geq 1$. In this section we show first that $R_{m}\left[\frac{1}{m}\right]$ is geometrically integral over $\hat{F}_{m}^{n r}$. Then we determine the structure of $\pi_{0}\left(M_{m} \times{ }_{\hat{F}^{n r}} \mathbb{C}_{\varpi}\right)$ together with the action of $G L_{n}(\mathfrak{o}) \times \mathfrak{o}_{B}^{\times} \times G_{\hat{F}^{n r}}$.

Denote as in Cor. 3.4 by $\hat{\mathfrak{o}}_{m}^{n r}$ the ring of integers in $\hat{F}_{m}^{n r}$, and let $\varpi_{m}$ be a uniformizer in $\hat{\mathfrak{o}}_{m}^{n r}$. Furthermore, we fix a set $\mathcal{R} \subset\left(\varpi^{-m} \mathfrak{o} / \mathfrak{o}\right)^{n}$ of representatives of the orbits of the action of $\left(\mathfrak{o} / \varpi^{m}\right)^{\times}$on

$$
\left(\varpi^{-m} \mathfrak{o} / \mathfrak{o}\right)^{n}-\left(\varpi^{-(m-1)} \mathfrak{o} / \mathfrak{o}\right)^{n} .
$$

Finally, we abbreviate the universal level- $m$-structure $\phi_{m}^{u n i v}$, cf. 2.2 , by $\phi_{m}$.

Proposition 4.2. (i) For $\alpha \in \mathcal{R}$ the element $\phi_{m}(\alpha) \in \mathfrak{m}_{R_{m}}$ of the regular local ring $R_{m}$ is irreducible. Moreover, the elements $\phi_{m}(\alpha)$ et $\phi_{m}(\beta)$ are not associated if $\alpha \neq \beta, \alpha, \beta \in \mathcal{R}$.

(ii) Up to a unit in $R_{m}$, the element $\varpi_{m}$ is equal to the product $\prod_{\alpha \in \mathcal{R}} \phi_{m}(\alpha)$.

(iii) The ring $R_{m} / \varpi_{m} R_{m}$ is reduced.

(iv) The ring $R_{m}\left[\frac{1}{\varpi}\right]$ is geometrically integral over $\hat{F}_{m}^{n r}$. 
Proof. (i) An element $\alpha \in \mathcal{R}$ can be completed to a basis $\alpha=\alpha_{1}, \ldots, \alpha_{n}$ of $\left(\varpi^{-m} \mathfrak{o} / \mathfrak{o}\right)^{n}$. By 2.2 we know that $\phi_{m}\left(\alpha_{1}\right), \ldots, \phi_{m}\left(\alpha_{n}\right)$ is a regular set of parameters of $R_{m}$. Hence, the element $\phi_{m}(\alpha)=\phi_{m}\left(\alpha_{1}\right)$ is in particular irreducible. Let $\alpha, \beta$ be two different elements of $\mathcal{R}$. For a point $x \in \operatorname{Spec}\left(R_{m}\right)$ consider the induced homomorphism

$$
\phi_{m, x}:\left(\varpi^{-m} \mathfrak{o} / \mathfrak{o}\right)^{n} \stackrel{\phi_{m}}{\longrightarrow} X^{\text {univ }}\left[\varpi^{m}\right]\left(R_{m}\right) \longrightarrow X^{\text {univ }}\left[\varpi^{m}\right](\kappa(x)) .
$$

If $h$ is the height of the connected part of the $\varpi$-divisible group $X^{\text {univ }}\left[\varpi^{\infty}\right] \otimes$ $\kappa(x)$, the kernel of $\phi_{m, x}$ is a direct summand of $\left(\varpi^{-m} \mathfrak{o} / \mathfrak{o}\right)^{n}$ of rank $h$ over $\mathfrak{o} /\left(\varpi^{m}\right)$. And conversely, if the rank of the kernel of $\phi_{m, x}$ is equal to $h$, then the height of the connected component of $X^{u n i v}\left[\varpi^{\infty}\right] \otimes \kappa(x)$ is $h$. Hence, if $\alpha$ and $\beta$ are two different elements of $\mathcal{R}$, the kernel of $\phi_{m, x}$ is at least of rank two if it contains $\alpha$ and $\beta$, and in this case the height of the connected component of $X^{u n i v}\left[\varpi^{\infty}\right] \otimes \kappa(x)$ is at least two. Now suppose that $\phi_{m}(\alpha)$ and $\phi_{m}(\beta)$ are associated prime elements. Let $x_{0} \in \operatorname{Spec}\left(R_{0}\right)$ correspond to the prime ideal $\varpi R_{0}$. Then, as we already stated in 2.3 , the height of the connected component of $X^{\text {univ }}\left[\varpi^{\infty}\right] \otimes \kappa\left(x_{0}\right)$ is one. As $R_{m}$ is finite and flat over $R_{0}$, there is a prime ideal $x_{m}$ of $R_{m}$ lying over $x_{0}$. Then the kernel of $\phi_{m, x_{m}}$ is a direct summand of rank one, hence generated by one element, $\gamma$ say. Let $g \in G L_{n}\left(\mathfrak{o} / \varpi^{m}\right)$ be an element with $g(\alpha)=\gamma$. Then $\alpha$ lies in the kernel of $\phi_{m, y_{m}}$, where $y_{m}=g\left(x_{m}\right)$, so $\phi_{m}(\alpha)$ is in the prime ideal corresponding to $y_{m}$. But if $\phi(\alpha)$ and $\phi(\beta)$ are associated, i.e. $\phi(\beta)=u \phi(\alpha)$ with a unit $u \in R_{m}$, we also have $\phi_{m, y_{m}}(\beta)=0$, and so the kernel of $\phi_{m, y_{m}}$ is at least of rank two, which cannot be, because $\phi_{m, y_{m}}=\phi_{m, x_{m}} \circ g$.

(ii) Let $\alpha \in \mathcal{R}$. Then $\mathfrak{p}=\phi(\alpha) R_{m}$ is a prime ideal, and because the kernel of $\phi_{m, \mathfrak{p}}$ contains $\alpha$, the connected component of $X^{\text {univ }}\left[\varpi^{\infty}\right] \otimes \kappa(\mathfrak{p})$ is at least of height one. Hence $\mathfrak{p}$ contains $\varpi$, and therefore $\varpi_{m}$ too. Because the elements $\phi_{m}(\alpha), \alpha \in \mathcal{R}$, are pairwise not associated, and because $R_{m}$ is a UFD, we see that

$$
\varpi_{m}=f \cdot \prod_{\alpha \in \mathcal{R}} \phi(\alpha)
$$

with some element $f \in R_{m}$. Now let us consider the unramified extension $F^{\prime}$ of $F$ of degree $n$ inside $\hat{F}^{n r}$. Denote by $\mathfrak{o}^{\prime}$ the ring of integers of $F^{\prime}$ and fix a formal $\mathfrak{o}^{\prime}$-module $X$ of $F^{\prime}$-height one. Let $\iota: \mathbb{X} \rightarrow X \times{ }_{\mathfrak{o}^{\prime}} \mathbb{F}$ be an isomorphism, such that the pair $\left(X \times_{\mathfrak{o}^{\prime}} \hat{\mathfrak{o}}^{n r}, \iota\right.$ ) is a deformation of $\mathbb{X}$ (as a formal $\mathfrak{o}$-module of height $n)$. This pair corresponds to a point $x$ in $\operatorname{Spec}\left(R_{0}\right)$. We lift this point to a point $y$ in $\operatorname{Spec}\left(R_{m}\right)$. The residue field $\kappa(y)$ at $y$ is then an extension of $F^{\prime}$ generated by the $\varpi^{m}$-torsion points of $X$. Denote by $v$ the valuation on this 
extension which is normalized by $v(\varpi)=1$. Then the valuation of a $\varpi^{m}$-torsion point of $X$, which is not annihilated by $\varpi^{m-1}$, is equal to $\frac{1}{\left(q^{n}-1\right) q^{n(m-1)}}$. Mapping $\varpi_{m}$ into $\kappa(y)$ and using the equation above we calculate

$$
\begin{aligned}
\frac{1}{(q-1) q^{m-1}} & =v\left(\varpi_{m}\right) \geq \sum_{\alpha \in \mathcal{R}} v\left(\phi_{m, y}(\alpha)\right)=\sum_{\alpha \in \mathcal{R}} \frac{1}{\left(q^{n}-1\right) q^{n(m-1)}} \\
& =\frac{\left(q^{n}-1\right) q^{n(m-1)}}{(q-1) q^{m-1}} \frac{1}{\left(q^{n}-1\right) q^{n(m-1)}}=\frac{1}{(q-1) q^{m-1}} .
\end{aligned}
$$

And this shows that $f$ is necessarily a unit in $R_{m}$.

(iii) This is an immediate consequence of (i) and (ii).

(iv) By [EGA], Cor. 18.9.8, it suffices to show that the fibres of

$$
\operatorname{Spec}\left(R_{m}\right) \longrightarrow \operatorname{Spec}\left(\hat{\mathfrak{o}}_{m}^{n r}\right)
$$

are geometrically reduced. By (iii), this is the case for the fibre over the closed point. Let us now consider the generic fibre. Let $E$ be a field containing $\hat{F}_{m}^{n r}$. Because $R_{m}\left[\frac{1}{\varpi}\right]$ is étale over $R_{0}\left[\frac{1}{\varpi}\right]$, the ring extension

$$
R_{0}\left[\frac{1}{\varpi}\right] \otimes_{\hat{F}^{n r}} E \rightarrow R_{m}\left[\frac{1}{\varpi}\right] \otimes_{\hat{F}^{n r}} E=\prod_{\sigma \in \operatorname{Gal}\left(\hat{F}_{m}^{n r} / \hat{F}^{n r}\right)} R_{m}\left[\frac{1}{\varpi}\right] \otimes_{\hat{F}_{m}^{n r}} E
$$

is étale too. Because $R_{0}\left[\frac{1}{\varpi}\right] \otimes_{\hat{F}^{n r}} E$ maps injectively into $E\left[\left[u_{1}, \ldots, u_{n-1}\right]\right]$, this ring is reduced. By general results on étale extensions,

$$
R_{m}\left[\frac{1}{\varpi}\right] \otimes_{\hat{F}_{m}^{n r}} E
$$

which is étale over $R_{0}\left[\frac{1}{\varpi}\right] \otimes_{\hat{F}^{n r}} E$, is reduced too. Therefore $R_{m}\left[\frac{1}{\varpi}\right] \otimes_{\hat{F}_{m}^{n r}} E$ is reduced.

Theorem 4.3. (i) Let $E$ be a finite separable extension of $\hat{F}^{n r}$, which contains the Lubin-Tate extension $\hat{F}_{m}^{n r}$ of $\hat{F}^{n r}$. Then the rigid-analytic space

$$
M_{m} \times_{\hat{F}^{n r}} \operatorname{Sp}(E)=\mathcal{M}_{m}^{r i g} \times_{\hat{F}^{n r}} \operatorname{Sp}(E)
$$


over $E$ has $(q-1) q^{m-1}$ connected components. These are the fibres of the morphism

$$
M_{m} \times_{\hat{F}^{n r}} \operatorname{Sp}(E) \longrightarrow \operatorname{Sp}\left(\hat{F}_{m}^{n r}\right) \times_{\hat{F}^{n r}} \operatorname{Sp}(E)=\operatorname{Sp}\left(\hat{F}_{m}^{n r} \otimes_{\hat{F}^{n r}} E\right)
$$

Proof. For the construction of the rigid-analytic space associated to a formal scheme we refer to $[\mathrm{dJ} 2]$, sec. 7 . The first assertion clearly follows from the second. Because

$$
M_{m} \times \hat{F}^{n r} \operatorname{Sp}(E)=\coprod_{\sigma \in \operatorname{Gal}\left(\hat{F}_{m}^{n r} / \hat{F}^{n r}\right)} M_{m} \times_{\hat{F}_{m}^{n r}} \operatorname{Sp}(E)
$$

we only need to show that $M_{m} \otimes_{\hat{F}_{m}^{n r}} \operatorname{Sp}(E)$ is connected. By [dJ2], 7.2.4 (g), we have

$$
M_{m} \otimes_{\hat{F}_{m}^{n r}} \operatorname{Sp}(E)=\operatorname{Spf}\left(R_{m} \hat{\otimes}_{\hat{\mathfrak{o}}_{m}^{n r}} \mathfrak{o}_{E}\right)^{r i g}
$$

where $\mathfrak{o}_{E}$ is the ring of integers in $E$. By [dJ2], 7.3.5, this space is connected if the ring

$$
R_{m} \hat{\otimes}_{\hat{\mathfrak{o}}_{m}^{n r} \mathfrak{o}_{E}}=R_{m} \otimes_{\hat{\mathfrak{o}}_{m}^{n r}} \mathfrak{o}_{E}
$$

(this equality holds because $E / \hat{F}_{m}^{n r}$ is finite) is integrally closed. This ring is contained in $R_{m} \otimes_{\hat{\mathfrak{o}}_{m}^{n r}} E=R_{m}\left[\frac{1}{\varpi}\right] \otimes_{\hat{F}_{m}^{n r}} E$ which is integral, by the preceding proposition 4.2. By [Bou], V, $\S 1.7$, Cor. to Prop. $19, R_{m} \otimes_{\hat{\mathfrak{o}}_{m}^{n r}} \mathfrak{o}_{E}$ is integrally closed.

Let $\mathbb{C}_{\varpi}$ be a completion of an algebraic closure $\bar{F}^{n r}$ of $\hat{F}^{n r}$. Denote by $G_{\hat{F}^{n r}}=$ $\operatorname{Gal}\left(\bar{F}^{n r} / \hat{F}^{n r}\right)$ the absolute Galois group of $\hat{F}^{n r}$. By continuity it acts on $\mathbb{C}_{\varpi}$. In the following we use the isomorphism $\hat{F}_{m}^{n r} \otimes_{\hat{F}^{n r}} \mathbb{C}_{\varpi} \simeq \prod_{\sigma \in \operatorname{Gal}\left(\hat{F}_{m}^{n r} / \hat{F}^{n r}\right)} \mathbb{C}_{\varpi}$ given by

$$
\lambda \otimes \mu \mapsto\left(\sigma^{-1}(\lambda) \mu\right)_{\sigma \in \operatorname{Gal}\left(\hat{F}_{m}^{n r} / \hat{F}^{n r}\right)}
$$

This isomorphism is used to identify the connected components of $\operatorname{Sp}\left(\hat{F}_{m}^{n r} \otimes_{\hat{F}^{n r}}\right.$ $\left.\mathbb{C}_{\varpi}\right)$ with the connected components of $\coprod_{\sigma \in \operatorname{Gal}\left(\hat{F}_{m}^{n r} / \hat{F}^{n r}\right)} \operatorname{Sp}\left(\mathbb{C}_{\varpi}\right)$ which we identify with its indexing set $\operatorname{Gal}\left(\hat{F}_{m}^{n r} / \hat{F}^{n r}\right)$. The latter group gets identified via the 
character $\chi_{m}$ with $\left(\mathfrak{o} / \varpi^{m}\right)^{\times}$. Note that if we let $\tau \in G_{\hat{F}^{n r}}$ act on the second factor of $\hat{F}_{m}^{n r} \otimes_{\hat{F}^{n r}} \mathbb{C}_{\varpi}$, we have, via the isomorphism above,

$$
\left(\left(\sigma^{-1}(\lambda) \tau(\mu)\right)_{\sigma}=\tau\left((\sigma \circ \tau)^{-1}(\lambda) \mu\right)_{\sigma}\right)=\tau\left(\left(\sigma^{-1}(\lambda) \mu\right)_{\sigma \circ \tau^{-1}}\right) .
$$

Therefore, on the indexing set $\operatorname{Gal}\left(\hat{F}_{m}^{n r} / \hat{F}^{n r}\right), \tau$ acts by multiplication by $\left(\left.\tau\right|_{\hat{F}_{m}^{n r}}\right)^{-1}$, and consequently on $\left(\mathfrak{o} / \varpi^{m}\right)^{\times}$by $\chi_{m}\left(\left.\tau\right|_{\hat{F}_{m}^{n r}}\right)^{-1}$.

Theorem 4.4. (i) The morphism $M_{m} \rightarrow \operatorname{Sp}\left(\hat{F}_{m}^{n r}\right)$ induces a bijection

$$
\pi_{0}\left(M_{m} \times_{\hat{F}^{n r}} \operatorname{Sp}\left(\mathbb{C}_{\varpi}\right)\right) \stackrel{\simeq}{\longrightarrow} \pi_{0}\left(\operatorname{Sp}\left(\hat{F}_{m}^{n r} \times_{\hat{F}^{n r}} \mathbb{C}_{\varpi}\right)\right),
$$

and the set on the right is identified with $\left(\mathfrak{o} / \varpi^{m}\right)^{\times}$, as explained above. The resulting bijection

$$
\pi_{0}\left(M_{m} \times_{\hat{F}^{n r}} \operatorname{Sp}\left(\mathbb{C}_{\varpi}\right)\right) \stackrel{\simeq}{\longrightarrow}\left(\mathfrak{o} / \varpi^{m}\right)^{\times}
$$
by

is $G L_{n}(\mathfrak{o}) \times \mathfrak{o}_{B}^{\times} \times G_{\hat{F}^{n r}}$-equivariant if we let $G L_{n}(\mathfrak{o}) \times \mathfrak{o}_{B}^{\times} \times G_{\hat{F}^{n r}}$ act on $\left(\mathfrak{o} / \varpi^{m}\right)^{\times}$

$$
(g, b, \tau) \mapsto \operatorname{det}(g) N r d(b)^{-1} \chi\left(\left.\tau\right|_{\hat{F}_{\infty}^{n r}}\right)^{-1} \quad \bmod \left(1+\varpi^{m} \mathfrak{o}\right)
$$

Here $N r d: \mathfrak{o}_{B}^{\times} \rightarrow \mathfrak{o}^{\times}$denotes the reduced norm.

(ii) In particular, the zero'th l-adic étale cohomology group decomposes as follows:

$$
H^{0}\left(M_{m} \times_{\hat{F}^{n r}} \operatorname{Sp}\left(\mathbb{C}_{\varpi}\right), \overline{\mathbb{Q}_{l}}\right) \simeq \bigoplus_{\omega}(\omega \circ \text { det }) \otimes(\omega \circ N r d)^{-1} \otimes\left(\omega \circ r e c_{\hat{F}^{n r}}\right)
$$

where $\omega:\left(\mathfrak{o} / \varpi^{m}\right)^{\times} \rightarrow \overline{\mathbb{Q}}_{l} \times$ runs through all $\overline{\mathbb{Q}_{l}}$-valued characters of $\left(\mathfrak{o} / \varpi^{m}\right)^{\times}$, and rec $_{\hat{F}^{n r}}$ is the reciprocity map from local class field theory (normalized such that an arithmetic Frobenius is mapped to a uniformizer). 
Proof. (i) As $R_{m}$ is normal, by [dJ2], 7.3.5., the rigid space $M_{m}=\operatorname{Spf}\left(R_{m}\right)^{\text {rig }}$ is connected. By [Co], Cor. 3.2.3, there is a finite separable extension $E$ of $\hat{F}^{n r}$ such that the canonical map

$$
M_{m} \times_{\hat{F}^{n r}} \operatorname{Sp}\left(\mathbb{C}_{\varpi}\right) \longrightarrow M_{m} \times_{\hat{F}^{n r}} \operatorname{Sp}(E)
$$

induces a bijection of the connected components:

$$
\pi_{0}\left(M_{m} \times_{\hat{F}^{n r}} \operatorname{Sp}\left(\mathbb{C}_{\varpi}\right)\right) \stackrel{\simeq}{\longrightarrow} \pi_{0}\left(M_{m} \times_{\hat{F}^{n r}} \operatorname{Sp}(E)\right)
$$

By theorem 4.3, we can take here $E=\hat{F}_{m}^{n r}$, and get:

$$
\begin{aligned}
\pi_{0}\left(M_{m} \times_{\hat{F}^{n r}} \operatorname{Sp}\left(\mathbb{C}_{\varpi}\right)\right) & =\pi_{0}\left(M_{m} \times_{\hat{F}^{n r}} \operatorname{Sp}\left(\hat{F}_{m}^{n r}\right)\right)=\pi_{0}\left(\operatorname{Sp}\left(\hat{F}_{m}^{n r} \otimes_{\hat{F}^{n r}} \hat{F}_{m}^{n r}\right)\right) \\
& =\pi_{0}\left(\operatorname{Sp}\left(\prod_{\sigma \in \operatorname{Gal}\left(\hat{F}_{m}^{n r} / \hat{F}^{n r}\right)} \hat{F}_{m}^{n r}\right)\right) \\
& =\operatorname{Gal}\left(\hat{F}_{m}^{n r} / \hat{F}^{n r}\right) \stackrel{\chi}{\longrightarrow}\left(\mathfrak{o} / \varpi^{m}\right)^{\times} .
\end{aligned}
$$

Here we used the isomorphism $\hat{F}_{m}^{n r} \otimes_{\hat{F}^{n r}} \hat{F}_{m}^{n r} \simeq \prod_{\sigma \in \operatorname{Gal}\left(\hat{F}_{m}^{n r} / \hat{F}^{n r}\right)} \hat{F}_{m}^{n r}$ given by

$$
\lambda \otimes \mu \mapsto\left(\sigma^{-1}(\lambda) \mu\right)_{\sigma \in \operatorname{Gal}\left(\hat{F}_{m}^{n r} / \hat{F}^{n r}\right)}
$$

The action of the Galois group $G_{K}$ on $R_{m}$ factors through $G L_{n}(\mathfrak{o})$, so let $g \in G L_{n}(\mathfrak{o})$ come from some $\rho \in G_{K}$. Then, for a $\varpi^{m}$-torsion point $a$ of $L T$ in $\hat{F}_{m}^{n r}$ we have

$$
\rho(a)=[\tilde{\chi}(\rho)]_{L T}(a) .
$$

But $\tilde{\chi}(\rho)$ is the element by which $\rho$ acts on the Tate module $\Lambda^{n}(T)$, by theorem 3.3. On the other hand, $g$ acts as $\operatorname{det}(g)$ on $\Lambda^{n}(T)$. Therefore, $g$ acts on the torsion point $a \in \hat{\mathfrak{o}}_{m}^{n r} \subset R_{m}$ by $[\operatorname{det}(g)]_{L T}(a)$. Via the above identification of $\pi_{0}\left(M_{m} \times \hat{F}^{n r} \operatorname{Sp}\left(\mathbb{C}_{\varpi}\right)\right)$ with $\left(\mathfrak{o} / \varpi^{m}\right)^{\times}$, we get that $G L_{n}(\mathfrak{o})$ acts on $\left(\mathfrak{o} / \varpi^{m}\right)^{\times}$via the determinant $\left(\bmod \left(1+\varpi^{m} \mathfrak{o}\right)\right)$. The action of $G_{\hat{F}^{n r}}$ we already computed above. Finally, we consider the action of $\mathfrak{o}_{B}^{\times}$. Let $\mathfrak{o}^{\prime} \subset \mathfrak{o}_{B}$ be the maximal unramified extension of $\mathfrak{o}$ in $\mathfrak{o}_{B}$. Because the reduced norm on $\mathfrak{o}_{B}^{\times}$, when restricted to $\left(\mathfrak{o}^{\prime}\right)^{\times}$, maps $\left(\mathfrak{o}^{\prime}\right)^{\times}$surjectively onto $\mathfrak{o}^{\times}$, it suffices to calculate the action of $\left(\mathfrak{o}^{\prime}\right)^{\times} \subset \mathfrak{o}_{B}^{\times}$. Let $X$ be a formal $\mathfrak{o}^{\prime}$-module of height one over $\mathfrak{o}^{\prime}$. Then $X \hat{\otimes}_{\mathfrak{o}^{\prime}} \hat{\mathfrak{o}}^{n r}$, when equipped with an isomorphism of its special fibre with $\mathbb{X}$, corresponds to a 
point $x$ in $\operatorname{Spec}\left(R_{0}\right)$ which is fixed by the action of $\left(\mathfrak{o}^{\prime}\right)^{\times}$. Let $y \in \operatorname{Spec}\left(R_{m}\right)$ be a point over $x$. Then $\mathfrak{o}_{m}^{\prime}:=R_{m} / y$ is the ring of integers in $\hat{F}^{n r} \cdot F_{m}^{\prime}$, where $F_{m}^{\prime}$ is the Lubin-Tate extension of $F^{\prime}=\mathfrak{o}^{\prime}\left[\frac{1}{\varpi}\right]$ generated by the $\varpi^{m}$-torsion points of $X$. By local class field theory, the action of an element $b \in\left(\mathfrak{o}^{\prime}\right)^{\times}$on the subfield $\hat{F}_{m}^{n r} \cap F_{m}^{\prime} \subset F_{m}^{\prime}$ is given by the norm of $N_{F^{\prime} / F}(b)=N r d(b)$ (on torsion points of $L T$ inside $\left.\hat{F}_{m}^{n r}\right)$. But the action of $b \in \mathfrak{o}_{B}^{\times}$on a triple $(X, \iota, \phi)$ is defined by $(X, \iota \circ b, \phi)$, and the latter object is equivalent to $\left(X, \iota, b^{-1} \circ \phi\right)$, because $X$ has multiplication by $\mathfrak{o}^{\prime}$. Hence the action of $b$ (via $R_{m}$ and specialisation) on the $\varpi^{m}$-torsion points of $X$ is given by $\left[b^{-1}\right]_{X}$. Therefore, it acts on the $\varpi^{m}$-torsion points of $L T$ by $\left[N r d(b)^{-1}\right]_{L T}$. Via the above identification of the connected components of $M_{m} \times \hat{F}^{n r} \operatorname{Sp}\left(\mathbb{C}_{\varpi}\right)$ with $\left(\mathfrak{o} / \varpi^{m}\right)^{\times}, b$ acts on the latter set by multiplication with $\operatorname{Nrd}(b)^{-1}$. This proves that $\mathfrak{o}_{B}^{\times}$acts on $\left(\mathfrak{o} / \varpi^{m}\right)^{\times}$by $N r d^{-1}$.

(ii) It is well known, that the local reciprocity map (normalized such that an arithmetic Frobenius is mapped to a uniformizer) fullfills

$$
r e c_{\hat{F}^{n r}}(\tau)=\chi(\tau)^{-1}
$$

From this and the first part of the theorem the assertion follows immediately.

We can finally also pass to the limit over all $m$ and get a natural $G L_{n}(\mathfrak{o}) \times$ $\mathfrak{o}_{B}^{\times} \times G_{\hat{F}^{n r}}$-equivariant map

$$
\underset{m}{\lim } \pi_{0}\left(M_{m} \times \hat{F}^{n r} \operatorname{Sp}\left(\mathbb{C}_{\varpi}\right)\right) \stackrel{\simeq}{\longrightarrow} \mathfrak{o}^{\times}
$$

where on the right side the group $G L_{n}(\mathfrak{o}) \times \mathfrak{o}_{B}^{\times} \times G_{\hat{F}^{n r}}$ acts by

$$
(g, b, \tau) \mapsto \operatorname{det}(g) N r d(b)^{-1} r e c_{\hat{F}^{n r}}(\tau) .
$$

And hence:

$$
\lim _{\vec{m}} H^{0}\left(M_{m} \times \hat{F}^{n r} \operatorname{Sp}\left(\mathbb{C}_{\varpi}\right), \overline{\mathbb{Q}_{l}}\right) \simeq \bigoplus_{\omega}(\omega \circ \operatorname{det}) \otimes(\omega \circ N r d)^{-1} \otimes\left(\omega \circ r e c_{\hat{F}^{n r}}\right)
$$

where $\omega$ runs through all continuous characters $\mathfrak{o}^{\times} \rightarrow \overline{\mathbb{Q}}_{l} \times$ (with the discrete topology on $\overline{\mathbb{Q}}_{l}^{\times}$). 


\section{REFERENCES}

[Bo] P. Boyer, Mauvaise réduction des variétés de Drinfeld et correspondance de Langlands locale. Invent. Math. 138 (1999), no. 3, 573-629.

[Bou] N. Bourbaki, Commutative algebra. Chapters 1-7. Elements of Mathematics. SpringerVerlag, Berlin, 1989.

[Ca] H. Carayol, Nonabelian Lubin-Tate theory. Automorphic forms, Shimura varieties, and $L$-functions, Vol. II (Ann Arbor, MI, 1988), 15-39, Perspect. Math., 11, Academic Press, Boston, MA, 1990.

[Ch] C.-L. Chai, The group action on the closed fiber of the Lubin-Tate moduli space. Duke Math. J. 82 (1996), no. 3, 725-754.

[Co] B. Conrad, Irreducible components of rigid spaces. Ann. Inst. Fourier (Grenoble) 49 (1999), no. 2, 473-541.

[D] V. G. Drinfeld, Elliptic modules. English translation: Math. USSR-Sb. 23 (1974), no. 4, 561-592.

[EGA] A. Grothendieck, J. Dieudonné, Éléments de géométrie algébrique. IV. Étude locale des schémas et des morphismes de schémas IV. Inst. Hautes Études Sci. Publ. Math. No. 32, 1967.

[F] G. Faltings, Group schemes with strict $\mathcal{O}$-action. Dedicated to Yuri I. Manin on the occasion of his 65th birthday. Mosc. Math. J. 2 (2002), no. 2, 249-279.

[Ha] M. Hazewinkel, Formal groups and applications. Pure and Applied Mathematics, 78. Academic Press, New York-London, 1978.

[HG] M. J. Hopkins, B. H. Gross, Equivariant vector bundles on the Lubin-Tate moduli space. Topology and representation theory (Evanston, IL, 1992), 23-88, Contemp. Math., 158, Amer. Math. Soc., Providence, RI, 1994.

[HT] M. Harris, R. Taylor, The geometry and cohomology of some simple Shimura varieties. With an appendix by Vladimir G. Berkovich. Annals of Mathematics Studies, 151. Princeton University Press, Princeton, NJ, 2001.

[dJ1] A. J. de Jong, Étale fundamental groups of non-Archimedean analytic spaces. Special issue in honour of Frans Oort. Compositio Math. 97 (1995), no. 1-2, 89-118.

[dJ2] A. J. de Jong, Crystalline Dieudonné module theory via formal and rigid geometry. Inst. Hautes Études Sci. Publ. Math. No. 82 (1995), 5-96.

[L] J. Lubin, Determinants for one-dimensional formal modules, unpublished manuscript.

[LT] J. Lubin, J. T. Tate, Formal moduli for one-parameter formal Lie groups. Bull. Soc. Math. France 94 (1966), 49-59.

[R] M. Raynaud, Schémas en groupes de type $(p, \ldots, p)$, Bull. Soc. Math. France, vol. 102 (1974), 241-280.

[SGA1] A. Grothendieck, Revêtements étales et groupe fondamental (SGA 1). Séminaire de géométrie algébrique du Bois Marie 1960-61. Directed by A. Grothendieck. With two papers by M. Raynaud. Updated and annotated reprint of the 1971 original. Documents Mathmatiques (Paris), 3. Société Mathématique de France, Paris, 2003.

[SGA2] A. Grothendieck, Cohomologie locale des faisceaux cohérents et théorèmes de Lefschetz locaux et globaux (SGA 2). Séminaire de géométrie algébrique du Bois Marie, 1962. Augmenté d'un exposé de Michèle Raynaud. With a preface and edited by Yves Laszlo. Revised reprint of the 1968 French original. Documents Mathématiques (Paris), 4. Société Mathématique de France, Paris, 2005.

[St] M. Strauch, Deformation spaces of one-dimensional formal modules and their cohomology. Preprint, Münster, 2006; available at: wwwmath1.uni-muenster.de/sfb/

[W] S. Wewers, Non-abelian Lubin-Tate theory via stable reduction. Preprint, 2006. 
[Y] T. Yoshida, On non-abelian Lubin-Tate theory via vanishing cycles. 2004, to appear in Annales de l'Institut Fourier.

Matthias Strauch

Department of Pure Mathematics and Mathematical Statistics

Centre for Mathematical Sciences, University of Cambridge

Wilberforce Road, Cambridge, CB3 0WB, United Kingdom

E-mail: M.Strauch@dpmms.cam.ac.uk 